\title{
A Model-Free Design of the Youla Parameter on the Generalized Internal Model Control Structure with Stability Constraint
}

\author{
Kazuhiro Yubai, Akitaka Mizutani and Junji Hirai \\ Mie University \\ Japan
}

\section{Introduction}

In the design of the control system, the plant perturbations and the plant uncertainties could cause the performance degradation and/or destabilization of the control system. The $H_{\infty}$ control synthesis and the $\mu$ synthesis are well known as the suitable controller syntheses for the plant with the large plant perturbations and/or the plant uncertainties (Zhou \& Doyle, 1998), and many successful applications are also reported in various fields. However, these controller syntheses provide the controller robustly stabilizing the closed-loop system for the worst-case and overestimated disturbances and uncertainties at the expense of the nominal control performance. It means that there exists a trade-off between the nominal control performance and the robustness in the design of the control system.

Meanwhile from the view point of the control architecture, the Generalized Internal Model Control (GIMC) structure is proposed by Zhou using Youla parameterization (Vidyasagar, 1985) to resolve the above-mentioned trade-off (Campos-Delgado \& Zhou, 2003; Zhou \& Ren, 2001). The GIMC structure is interpreted as an extension of the Internal Model Control (IMC) (Morari \& Zafiriou, 1997), which is only applicable to stable plants, to unstable plants by introducing coprime factorization. The GIMC structure consists of a conditional feedback structure and an outer-loop controller. The conditional feedback structure can detect model uncertainties and any disturbances, and they are compensated through the Youla parameter. It means that the robustness of the control system in the GIMC structure is specified by the Youla parameter. On the other hand, in case where there exist no plant uncertainties and no disturbances, the conditional feedback structure would detect nothing, and the feedback control system would be governed only by the outer-loop controller. Since the nominal control performance is independent of the Youla parameter, the outer-loop controller can be designed according to various controller design techniques, and the trade-off between the nominal control performance and the robustness is resolved.

For the design of the Youla parameter, we proposed the design method using the dual Youla parameter which represents the plant perturbation and/or the plant uncertainties (Matsumoto et al., 1993; Yubai et al., 2007). The design procedure is as follows: The dual Youla parameter is identified by the Hansen scheme (Hansen et al., 1989) using appropriate identification techniques, and the Youla parameter is designed based on the robust controller 
synthesis. However, since it is difficult to give a physical interpretation to the dual Youla parameter in general, we must select the weighting function for identification and the order of the identified model by trial and error. For implementation aspect, a low-order controller is much preferable, which means that a low-order model of the dual Youla parameter should be identified. However, it is difficult to identify the low-order model of the dual Youla parameter which contains enough information on the actual dual Youla parameter to design the appropriate Youla parameter. Moreover, there may be the cases where an accurate and reasonably low-order model of the dual Youla parameter can not be obtained easily.

To avoid these difficulties in system identification of the dual Youla parameter, this article addresses the design method of the Youla parameter by model-free controller synthesis. Model-free controller syntheses have the advantages that the controller is directly synthesized or tuned only from the input/output data collected from the plant, and no plant mathematical model is required for the controller design, which avoids the troublesome model identification of the dual Youla parameter. Moreover, since the order and the controller structure are specified by the designer, we can easily design a low-order Youla parameter by model-free controller syntheses.

A number of model-free controller syntheses have been proposed, e.g., the Iterative Feedback Tuning (IFT) (Hjalmarsson, 1998), the Virtual Reference Feedback Tuning (VRFT) (Campi et al., 2002), and the Correlation-based Tuning (CbT) (Miskovic et al., 2007) and so on. These model-free controller syntheses address the model matching problem as a typical control objective. Since the IFT and the CbT basically deal with nonlinear optimization problems, they require the iterative experiments to update the gradient of the cost function and the Hessian for the Gauss-Newton method at each iterative parameter update. On the other hand, the VRFT brings controllers using only a single set of input/output data collected from the plant if the controllers are linearly parameterized with respect to the parameter vector to be tuned. This article adopts the VRFT to design the Youla parameter to exploit the above-mentioned feature. However, the model-free controller syntheses have a common disadvantage that the stability of the closed-loop system can not be evaluated in advance of controller implementation because we have no mathematical plant model to evaluate the stability and/or the control performance. From the view point of safety, destabilization of the control system is not acceptable. Recently, the data-driven test on the closed-loop stability before controller implementation (Karimi et al., 2007; Yubai et al., 2011) and the data-driven controller synthesis at least guaranteeing the closed-loop stability (Heusden et al., 2010) are developed for the standard unity feedback control structure.

This article derives the robust stability condition for the design of the Youla parameter, and its sufficient condition is described as the $H_{\infty}$ norm of the product of the Youla and the dual Youla parameters. Moreover, the $H_{\infty}$ norm is estimated using the input/output data collected from the plant in the closed-loop manner. This sufficient condition of the robust stability is imposed as the stability constraint to the design problem of the Youla parameter based on the VRFT previously proposed by the authors (Sakuishi et al., 2008). Finally, the Youla parameter guaranteeing the closed-loop stability is obtained by solving the convex optimization.

The discussion is limited to SISO systems in this article. 


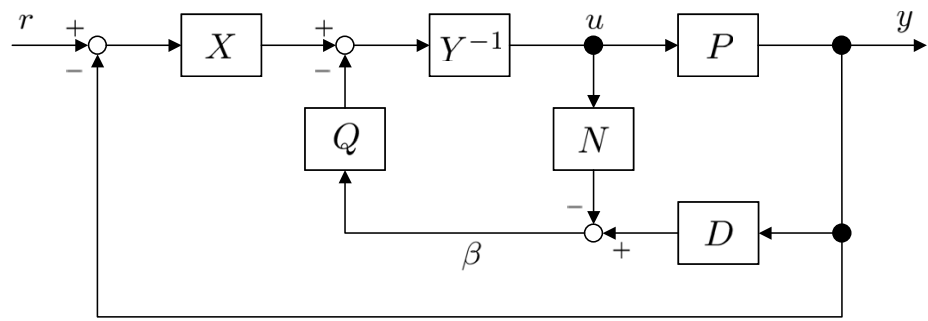

Fig. 1. GIMC structure.

\section{Robust control by the GIMC structure}

This section gives a brief review of the GIMC (Generalized Internal Model Control) structure and it is a control architecture solving the trade-off between the control performance and the robustness.

\subsection{GIMC structure}

A linear time-invariant plant $P_{0}$ is assumed to have a coprime factorization (Vidyasagar, 1985) on $\mathcal{R} \mathcal{H}_{\infty}$ as

$$
P_{0}=N D^{-1}, \quad N, D \in \mathcal{R} \mathcal{H}_{\infty},
$$

where $\mathcal{R} \mathcal{H}_{\infty}$ denotes the set of all real rational proper stable transfer functions. A nominal controller $C_{0}$ stabilizing $P_{0}$ is also assumed to have a coprime factorization on $\mathcal{R} \mathcal{H}_{\infty}$ as

$$
C_{0}=X Y^{-1}, \quad X, Y \in \mathcal{R} \mathcal{H}_{\infty},
$$

where $X$ and $Y$ satisfy the Bezout identity $X N+Y D=1$. Then a class of all stabilizing controllers $C$ is parameterized as (3), which is called as Youla parameterization, by introducing the Youla parameter $Q \in \mathcal{R} \mathcal{H}_{\infty}$ (Vidyasagar, 1985):

$$
C=(Y-Q N)^{-1}(X+Q D),
$$

where $Q$ is a free parameter and is determined arbitrarily as long as

$$
\operatorname{det}(Y(\infty)-Q(\infty) N(\infty)) \neq 0 .
$$

Then, the GIMC structure is constructed as Fig. 1 by using (1) and (3), where $r, u, y$ and $\beta$ represent reference inputs, control inputs, observation outputs and residual signals, respectively. The only difference between the GIMC structure and a standard unity feedback control structure shown in Fig. 2 is that the input of $D$ is $y$ in the GIMC structure instead of $e$. Since the GIMC structure has a conditional feedback structure, the Youla parameter $Q$ is only activated in the case where disturbances are injected and/or there exist plant uncertainties. If there is no disturbance and no plant uncertainty $(\beta=0), Q$ in the GIMC structure does not generate any compensation signals and the control system is governed by only a nominal controller $C_{0}$. It means that the nominal control performance is specified by only the nominal controller $C_{0}$. On the other hand, if there exist disturbances and/or plant uncertainties $(\beta \neq 0)$, 


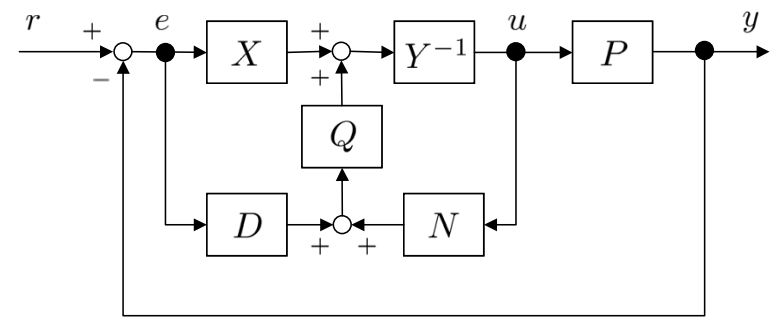

Fig. 2. A unity feedback control structure.

the inner loop controller $Q$ generates the compensation signal to suppress the effect of plant uncertainties and disturbances in addition to the nominal controller $C_{0}$.

In this way, the role of $C_{0}$ and that of $Q$ are clearly separated: $C_{0}$ could be designed to achieve the higher nominal control performance, while $Q$ could be designed to attain the higher robustness for plant uncertainties and disturbances. This is the reason why the GIMC structure is one of promising control architectures which solve the trade-off between the nominal control performance and the robustness in the design of the feedback control system. In this article, we address the design problem of the Youla parameter $Q$ using the input/output data set to generate an appropriate compensation signal to reduce the effect of plant uncertainties and/or disturbances on the assumption that the nominal controller $C_{0}$ which meets the given nominal control performance requirements has been already available.

\subsection{Dual Youla parameterization and robust stability condition}

For appropriate compensation of plant uncertainties, information on plant uncertainties is essential. In the design of the Youla parameter $Q$, the following parameterization plays an important role. On the assumption that the nominal plant $P_{0}$ factorized as (1) and its deviated version, $P$, are stabilized by the nominal controller $C_{0}$, then $P$ is parameterized by introducing a dual Youla parameter $R \in \mathcal{R} \mathcal{H}_{\infty}$ as follows:

$$
P=(N+Y R)(D-X R)^{-1} .
$$

This parameterization is called as the dual Youla parameterization, which is a dual version of the Youla parameterization mentioned in the previous subsection. It says that the actual plant $P$, which is deviated from the nominal plant $P_{0}$, can be represented by the dual Youla parameter $R$. By substituting (4) to the block-diagram shown by Fig. 1, we obtain the equivalent block-diagram shown by Fig. 3. From this block-diagram, the robust stability condition when the controlled plant deviates from $P_{0}$ to $P$ is derived as

$$
(1+R Q)^{-1} \in \mathcal{R} \mathcal{H}_{\infty}
$$

We must design $Q$ so as to meet this stability condition.

\section{Direct design of the Youla parameter from experimental data}

As stated in the previous subsection, the role of $Q$ is to suppress plant variations and disturbances. This article addresses the design problem of $Q$ to approach the closed-loop performance from $r$ to $y$, denoted by $G_{r y}$, to the its nominal control performance as an 


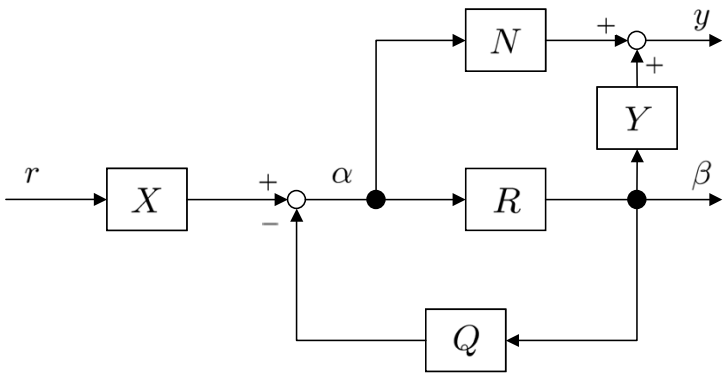

Fig. 3. Equivalent block-diagram of GIMC.

example. This design problem is formulated in frequency domain as a model matching problem;

$$
Q=\arg \min _{\tilde{Q}} J_{M R}(\tilde{Q}),
$$

where

$$
J_{\mathrm{MR}}(Q)=\left\|W_{M}\left(M-\frac{(N+R Y) X}{1+R Q}\right)\right\|_{2}^{2} .
$$

$M$ is a reference model for $G_{r y}$ given by the designer and it corresponds to the nominal control performance. $W_{M}$ is a frequency weighting function.

According to the model-based controller design techniques, the following typical controller design procedure is taken place: Firstly, we identify the dual Youla parameter $R$ using the input/output data set. Secondly, the Youla parameter $Q$ is designed based on the identified model of $R$. However, since the dual Youla parameter $R$ is described as

$$
R=D\left(P-P_{0}\right)\left\{Y\left(1+P C_{0}\right)\right\}^{-1},
$$

it depends on the coprime factors, $N, D, X$ and $Y$, which makes it difficult to give a physical interpretation for $R$. As a result, the identification of $R$ requires trial-and-error for the selection of the structure and/or the order of $R$. As is clear from (8), $R$ should be modeled as a high order model, the designed $Q$ tends to be a high order controller, which is a serious problem for implementation.

In this article, we address the direct design problem of the fixed-order and fixed-structural $Q$ from the input/output data set minimizing the evaluation function (7) without any model identification of $R$.

\subsection{Review of the Virtual Reference Feedback Tuning (VRFT)}

The Virtual Reference Feedback Tuning (VRFT) is one of model-free controller design methods to achieve the model matching. The VRFT provides the controller parameters using only the input/output data set so that the actual closed-loop property approaches to its reference model given by the designer. In this subsection, the basic concept and its algorithm are reviewed. 


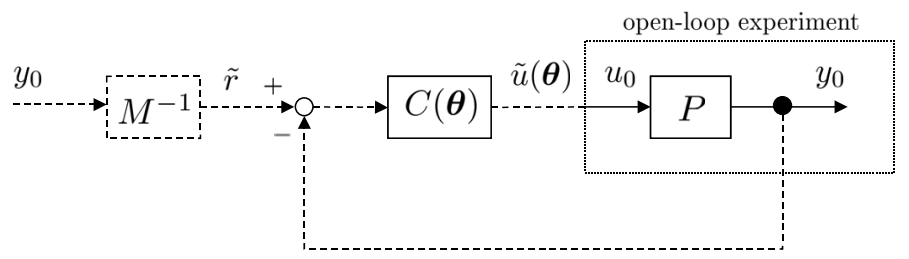

Fig. 4. Basic concept of the VRFT.

The basic concept of the VRFT is depicted in Fig. 4. For a stable plant, assume that the input/output data set $\left\{u_{0}(t), y_{0}(t)\right\}$ of length $N$ has been already collected in open-loop manner. Introduce the virtual reference $\tilde{r}(t)$ such that

$$
y_{0}(t)=M \tilde{r}(t),
$$

where $M$ is a reference model to be achieved. Now, assume that the output of the feedback system consisting of $P$ and $C(\boldsymbol{\theta})$ parameterized by the parameter vector $\boldsymbol{\theta}$ coincides with $y_{0}(t)$ when the virtual reference signal $\tilde{r}(t)$ is given as a reference signal. Then, the output of $C(\boldsymbol{\theta})$, denoted by $\tilde{u}(t, \theta)$ is represented as

$$
\begin{aligned}
\tilde{u}(t, \boldsymbol{\theta}) & =C(\boldsymbol{\theta})\left(\tilde{r}(t)-y_{0}(t)\right) \\
& =C(\boldsymbol{\theta})\left(M^{-1}-1\right) y_{0}(t) .
\end{aligned}
$$

If $\tilde{u}(t, \theta)=u_{0}(t)$, then the model matching is achieved, i.e.,

$$
M=\frac{P C(\boldsymbol{\theta})}{1+P C(\boldsymbol{\theta})} .
$$

Since the exact model matching is difficult in practice due to the restricted structural controller, the measurement noise injected to the output etc., we consider the alternative optimization problem:

$$
\hat{\boldsymbol{\theta}}=\arg \min _{\boldsymbol{\theta}} J_{\mathrm{VR}}^{N}(\boldsymbol{\theta})
$$

where

$$
\begin{aligned}
J_{\mathrm{VR}}^{N}(\boldsymbol{\theta}) & =\frac{1}{N} \sum_{t=1}^{N}\left[L\left(u_{0}(t)-\tilde{u}(t, \boldsymbol{\theta})\right)\right]^{2} \\
& =\frac{1}{N} \sum_{t=1}^{N}\left[L u_{0}(t)-C(\boldsymbol{\theta}) L\left(\tilde{r}(t)-y_{0}(t)\right)\right]^{2}
\end{aligned}
$$

$L$ is a prefilter given by the designer. By selection of $L=W_{M} M(1-M), \hat{\theta}$ would be a good approximation of the exact solution of the model matching problem $\overline{\boldsymbol{\theta}}$ even if $\tilde{u}(t, \boldsymbol{\theta}) \neq u_{0}(t)$ (Campi et al., 2002). Especially, in case where the controller $C(\boldsymbol{\theta})$ is linearly parameterized with respect to $\boldsymbol{\theta}$ using an appropriate transfer matrix $\boldsymbol{\sigma}$, i.e., $C(\boldsymbol{\theta})=\sigma^{\mathrm{T}} \boldsymbol{\theta}$, the optimal solution 
$\hat{\boldsymbol{\theta}}$ is calculated by the least-squares method as

$$
\hat{\boldsymbol{\theta}}=\left[\sum_{t=1}^{N} \boldsymbol{\varphi}(t) \boldsymbol{\varphi}^{\mathrm{T}}(t)\right]^{-1} \sum_{t=1}^{N} \boldsymbol{\varphi}(t) u_{L}(t),
$$

where $\varphi(t)=L \sigma\left(\tilde{r}(t)-y_{0}(t)\right), u_{L}(t)=L u_{0}(t)$.

\subsection{Direct tuning of $Q$ from experimental data by the VRFT}

This subsection describes the application of the VRFT to the design of the Youla parameter $Q$ without any model identification of the dual Youla parameter $R$. The experimental data set used in the controller design, $\left\{r_{0}(t), u_{0}(t), y_{0}(t)\right\}$, is collected from the closed-loop system composed of the perturbed plant $P$ and the nominal controller $C_{0}$. Define the Youla parameter $Q(z, \theta)$ linearly parameterized with respect to $\theta$ as

$$
Q(z, \boldsymbol{\theta})=\boldsymbol{\sigma}(z)^{\mathrm{T}} \boldsymbol{\theta},
$$

where $\sigma(z)$ is a discrete-time transfer function vector defined as

$$
\sigma(z)=\left[\sigma_{1}(z), \sigma_{2}(z), \cdots, \sigma_{n}(z)\right]^{\mathrm{T}},
$$

and $\theta$ is a parameter vector of length $n$ defined as

$$
\boldsymbol{\theta}=\left[\theta_{1}, \theta_{2}, \cdots, \theta_{n}\right]^{\mathrm{T}} .
$$

Then the model matching problem formulated as (6) can be rewritten with respect to $\theta$ as

$$
\overline{\boldsymbol{\theta}}=\arg \min _{\boldsymbol{\theta}} J_{\mathrm{MR}}(\boldsymbol{\theta}),
$$

where

$$
J_{\mathrm{MR}}(\boldsymbol{\theta})=\left\|W_{M}\left(M-\frac{(N+R Y) X}{1+R Q(\boldsymbol{\theta})}\right)\right\|_{2}^{2} .
$$

Under the condition that the dual Youla parameter $R$ is unknown, we will obtain the minimizer $\overline{\boldsymbol{\theta}}$ of $J_{\mathrm{MR}}(\boldsymbol{\theta})$ using the closed-loop experimental data set $\left\{r_{0}(t), u_{0}(t), y_{0}(t)\right\}$.

Firstly, we obtain the input and the output data of $R$ denoted by $\alpha(t)$, and $\beta(t)$, respectively. In Fig. 1, we treat the actual plant $P$ as the perturbed plant described by (4) and set $Q=0$ since $Q$ is a parameter to be designed. Then, we calculate $\alpha(t)$ and $\beta(t)$ using the input/output data, $\left\{u_{0}(t), y_{0}(t)\right\}$ collected from the plant when the appropriate reference signal $r_{0}(t)$ is applied to the standard unity feedback control structure as shown in Fig. 5. The signals $\alpha(t)$ and $\beta(t)$ are calculated as follows:

$$
\begin{aligned}
\alpha(t) & =X y_{0}(t)+Y u_{0}(t) \\
& =X r_{0}(t), \\
\beta(t) & =D y_{0}(t)-N u_{0}(t) .
\end{aligned}
$$

Although $\alpha(t)$ is an internal signal of the feedback control system, $\alpha(t)$ is an function of the external signal $r_{0}(t)$ given by the designer as is clear from (14). This means that the 
loop-gain from $\beta$ to $\alpha$ is equivalent to 0 , and that the input-output characteristic from $\beta$ to $\alpha$ is an open-loop system, which is also understood by Fig. 3 with $Q=0$. Moreover, since $R$ belongs to $\mathcal{R} \mathcal{H}_{\infty}$ according to the dual Youla parameterization, the input/output data set of $R$ is always available by an open-loop experiment. As a result, the basic requirement for the VRFT is always satisfied in this parameterization.

Secondly, we regard $y_{0}(t)$ as the output of the reference model $M$, and obtain the virtual reference $\tilde{r}(t)$ such that

$$
y_{0}(t)=M \tilde{r}(t)
$$

If there exists the parameter $\boldsymbol{\theta}$ such that $\alpha(t)=X \tilde{r}(t)-Q(\boldsymbol{\theta}) \beta(t)$, the exact model matching is achieved $\left(G_{r y}=M\right)$. According to the concept of the VRFT, the approximated solution of the model matching problem, $\hat{\boldsymbol{\theta}}$, is obtained by solving the following optimization problem:

$$
\hat{\boldsymbol{\theta}}=\arg \min _{\boldsymbol{\theta}} J_{\mathrm{VR}}^{N}(\boldsymbol{\theta}),
$$

where

$$
J_{\mathrm{VR}}^{N}(\boldsymbol{\theta})=\frac{1}{N} \sum_{t=1}^{N}\left[L_{M}(\alpha(t)-X \tilde{r}(t)+Q(\boldsymbol{\theta}) \beta(t))\right]^{2} .
$$

Since $Q(\boldsymbol{\theta})$ is linear with respect to the parameter vector $\boldsymbol{\theta}$ as defined in $(9), J_{\mathrm{VR}}^{N}(\boldsymbol{\theta})$ is rewritten as

$$
J_{\mathrm{VR}}^{N}(\boldsymbol{\theta})=\frac{1}{N} \sum_{t=1}^{N}\left[\boldsymbol{y}_{L}(t)-\boldsymbol{\varphi}(t)^{\mathrm{T}} \boldsymbol{\theta}\right]^{2},
$$

where

$$
\begin{aligned}
\boldsymbol{\varphi}(t) & =-L_{M} \sigma \beta(t), \\
y_{L}(t) & =L_{M}(\alpha(t)-X \tilde{r}(t)) .
\end{aligned}
$$

The minimizer of $J_{\mathrm{VR}}^{N}(\boldsymbol{\theta})$ is then calculated using the least-squares method as

$$
\hat{\boldsymbol{\theta}}=\left[\sum_{t=1}^{N} \boldsymbol{\varphi}(t) \boldsymbol{\varphi}^{\mathrm{T}}(t)\right]^{-1} \sum_{t=1}^{N} \boldsymbol{\varphi}(t) \boldsymbol{y}_{L}(t) .
$$

The filter $L_{M}$ is specified by the designer. By selecting $L_{M}=W_{M} M Y \Phi_{\alpha}(\omega)^{-1}, \hat{\boldsymbol{\theta}}$ could be a good approximation of $\overline{\boldsymbol{\theta}}$ in case $N \rightarrow \infty$, where $\Phi_{\alpha}(\omega)$ is a spectral density function of $\alpha(t)$. Moreover, this design approach needs an inverse system of the reference model, $M^{-1}$, when $\tilde{r}(t)$ is generated. However, by introducing $L_{M}$, we can avoid overemphasis by derivation in $M^{-1}$ in the case where the noise corrupted data $y_{0}(t)$ is used.

\subsection{Stability constraint on the design of $Q$ by the VRFT}

The design method of $Q$ based on the VRFT stated in the previous subsection does not explicitly address the stability issue of the resulting closed-loop system. Therefore, we can not evaluate whether the resulting Youla parameter $Q(\theta)$ actually stabilizes the closed-loop 


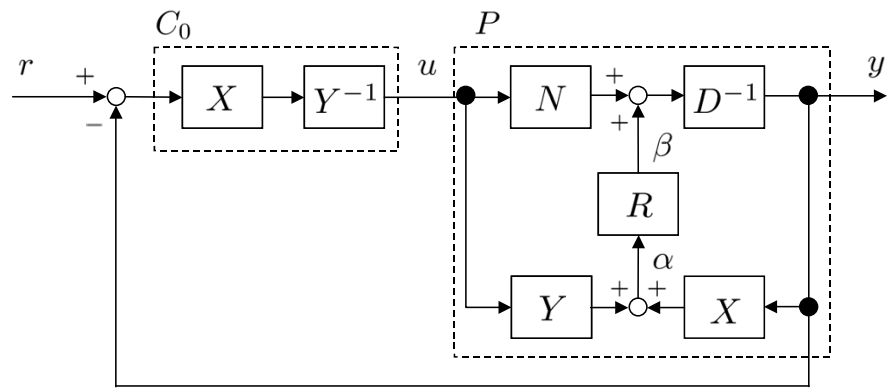

Fig. 5. Data acquisition of $\alpha(t)$ and $\beta(t)$.

system or not in advance of its implementation. To avoid the instability, the data-based stability constraint should be introduced in the optimization problem (17).

As stated in the subsection 2.2, the robust stability condition when the plant perturbs from $P_{0}$ to $P$ is described using $R$ and $Q$ as (5). However, (5) is non-convex with respect to the parameter $\theta$, and it is difficult to incorporate this stability condition into the least-squares based VRFT as the constraint. Using the small-gain theorem, the sufficient condition of the robust stability is derived as

$$
\delta=\|R Q(\boldsymbol{\theta})\|_{\infty}<1 .
$$

The alternative constraint (20) is imposed instead of (5), and the original constrained optimization problem is reduced to the tractable one (Matsumoto et al., 1993). Since model information on the plant can not be available in the model-free controller syntheses such as the VRFT, we must evaluate (20) using only the input/output data set $\left\{u_{0}(t), y_{0}(t)\right\}$ obtained from the closed-loop system. As is clear from Fig. 3, since the input and the output data of $R$ are $\alpha(t)$ and $\beta(t)$, respectively, the open-loop transfer function from $\alpha$ to $\xi(\theta)$ corresponds to $R Q(\boldsymbol{\theta})$ by introducing the virtual signal $\xi(t, \boldsymbol{\theta})=Q(\boldsymbol{\theta}) \beta(t)$. Assuming that $\alpha(t)$ is a $p$ times repeating signal of a periodic signal with a period $T$, i.e., $\alpha(t)$ is of length $N=p T$, the $H_{\infty}$ norm of $R Q(\boldsymbol{\theta})$ denoted by $\delta(\boldsymbol{\theta})$ can be estimated via the spectral analysis method as the ratio between the power spectral density function of $\alpha(t)$, denoted by $\Phi_{\alpha}\left(\omega_{k}\right)$, and the power cross spectral density function between $\alpha(t)$ and $\xi(t, \theta)$, denoted by $\Phi_{\alpha \xi}\left(\omega_{k}\right)$ (Ljung, 1999).

From the Wiener-Khinchin Theorem, $\Phi_{\alpha}\left(\omega_{k}\right)$ is represented as a discrete Fourier transform (DFT) of an auto-correlation of $\alpha(t)$, denoted by $R_{\alpha}(\tau)$ :

$$
\Phi_{\alpha}\left(\omega_{k}\right)=\frac{1}{T} \sum_{\tau=0}^{T-1} R_{\alpha}(\tau) e^{-i \tau \omega_{k}},
$$

where

$$
R_{\alpha}(\tau)=\frac{1}{T} \sum_{\tau=1}^{T-1} \alpha(t-\tau) \alpha(t)
$$

$\omega_{k}=2 \pi k /\left(T T_{s}\right)(T=0, \cdots,(T-1) / 2)$, and $T_{s}$ is a sampling time. The frequency points $\omega_{k}$ must be defined as a sequence with a much narrow interval for a good estimate of $\delta(\boldsymbol{\theta})$. 
A shorter sampling time $T_{S}$ is preferable to estimate $\delta(\theta)$ in higher frequencies, and a longer period $T$ improves the frequency resolution.

Similarly, $\Phi_{\alpha \xi}\left(\omega_{k}, \boldsymbol{\theta}\right)$ is estimated as a DFT of the cross-correlation between $\alpha(t)$ and $\xi(t, \boldsymbol{\theta})$, denoted by $R_{\alpha \xi}(\tau)$ :

$$
\hat{\Phi}_{\alpha \xi}\left(\omega_{k}, \boldsymbol{\theta}\right)=\frac{1}{T} \sum_{\tau=0}^{T-1} \hat{R}_{\alpha \xi}(\tau, \boldsymbol{\theta}) e^{-i \tau \omega_{k}},
$$

where

$$
\hat{R}_{\alpha \xi}(\tau, \boldsymbol{\theta})=\frac{1}{N} \sum_{\tau=1}^{N} \alpha(t-\tau) \xi(t, \boldsymbol{\theta}) .
$$

Using the $p$-periods cyclic signal $\alpha(t)$ in the estimate of $\hat{R}_{\alpha \tau}(\tau, \boldsymbol{\theta})$, the effect of the measurement noise involved in $\xi(t, \theta)$ is averaged and the estimate error in $\Phi_{\alpha \xi}\left(\omega_{k}, \theta\right)$ is then reduced. Especially, the measurement noise is normalized, the effect on the estimate of $\Phi_{\alpha \xi}\left(\omega_{k}, \theta\right)$ by the measurement noise is asymptotically reduced to 0 .

Since $Q(\theta)$ is linearly defined with respect to $\theta, \hat{R}_{\alpha \xi}(\tau, \theta)$ and $\hat{\Phi}_{\alpha \xi}\left(\omega_{k}, \theta\right)$ are also linear with respect to $\theta$. As a result, the stability constraint of (20) is evaluated using only the input/output data as

$$
\hat{\delta}(\boldsymbol{\theta})=\max _{\left\{\omega_{k} \mid \Phi_{\alpha}\left(\omega_{k}\right) \neq 0\right\}}\left|\frac{\hat{\Phi}_{\alpha \tilde{\xi}}\left(\omega_{k}, \boldsymbol{\theta}\right)}{\Phi_{\alpha}\left(\omega_{k}\right)}\right|<1 .
$$

Since this constraint is convex with respect to $\theta$ at each frequency point $\omega_{k}$, we can integrate this $H_{\infty}$ norm constraint into the optimization problem (17) and solve it as a convex optimization problem.

\subsection{Design algorithm}

This subsection describes the design algorithm of $Q(\theta)$ imposing the stability constraint.

[step 1] Collect the input/output data set $\left\{u_{0}(t), y_{0}(t)\right\}$ of length $N$ in the closed-loop manner in the unity feedback control structure shown in Fig. 5 when the appropriate reference signal $r_{0}(t)$ is applied.

[step 2] Calculate $\alpha(t)$ and $\beta(t)$ using the data set $\left\{r_{0}(t), u_{0}(t), y_{0}(t)\right\}$ as

$$
\begin{aligned}
& \alpha(t)=X r_{0}(t), \\
& \beta(t)=D y_{0}(t)-N u_{0}(t) .
\end{aligned}
$$

[step 3] Generate the virtual reference $\tilde{r}(t)$ such that

$$
y_{0}(t)=M \tilde{r}(t)
$$

[step 4] Solve the following convex optimization problem;

$$
\hat{\boldsymbol{\theta}}=\arg \min _{\boldsymbol{\theta}} J_{\mathrm{VR}}^{N}(\boldsymbol{\theta}),
$$




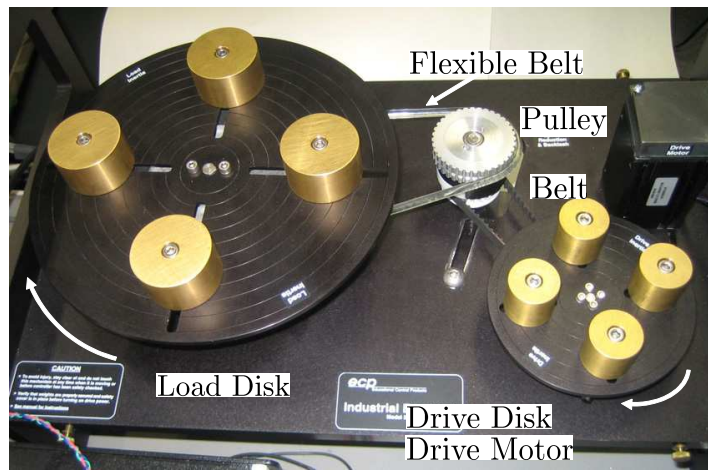

Fig. 6. Experimental set-up of a belt-driven two-mass system.

subject to

$$
\begin{aligned}
& \left|\frac{1}{T} \sum_{\tau=0}^{T-1} \hat{R}_{\alpha \mathcal{\xi}}(\tau, \theta) e^{-i \tau \omega_{k}}\right|<\left|\frac{1}{T} \sum_{\tau=0}^{T-1} R_{\alpha}(\tau) e^{-i \tau \omega_{k}}\right|, \\
& \omega_{k}=2 \pi k / T, \quad k=0, \ldots,(T-1) / 2 .
\end{aligned}
$$

\section{Design example}

To verify the effectiveness of the proposed design method, we address a velocity control problem of a belt-driven two-mass system frequently encountered in many industrial processes.

\subsection{Controlled plant}

The plant to be controlled is depicted as Fig. 6. The velocity of the drive disk is controlled by the drive motor connected to the drive disk. The pulley is connected to the load disk through the flexible belt, the restoring force of the flexible belt affects the velocity of the drive disk, which causes the resonant vibration of the drive disk. The resonant frequency highly depends on the position and the number of the weights mounted on the drive disk and the load disk. We treat this two-mass resonant system as the controlled plant $P$. Since the position and the number of the weights mainly changes the resonant frequency, a rigid model is treated as the nominal plant $P_{0}$ identified easily, which changes little in response to load change. The nominal plant $P_{0}$ is identified by the simple frequency response test as

$$
P_{0}=\frac{4964}{s^{2}+136.1 s+8.16} .
$$

Moreover, the delay time of $14 \mathrm{~ms}$ is emulated by the software as the plant perturbation in $P$, but it is not reflected in $P_{0}$. Due to the delay time, the closed-loop system tends to be destabilized when the gain of the feedback controller is high. This means that if the reference model with the high cut-off frequency is given, the closed-loop system readily destabilized. 


\subsection{Experimental condition}

For the simplicity, the design problem is restricted to the model matching of $G_{r y}$ approaching to its reference model $M$ in the previous section. However, the proposed method readily address the model matching of multiple characteristics. In the practical situations, we must solve the trade-off between several closed-loop properties. In this experimental set-up, we show the design result of the simultaneous optimization problem approaching the tracking performance, $G_{r y}$, and the noise attenuation performance, $G_{n y}$ to their reference models, $M$ and $T$, respectively. The evaluation function is defined as

$$
J_{\mathrm{MR}}(\boldsymbol{\theta})=\left\|W_{M}\left(M-\frac{(N+R Y) X}{1+R Q(\boldsymbol{\theta})}\right)\right\|_{2}^{2}+\left\|W_{T}\left(T-\frac{(N+R Y)(X+Q(\boldsymbol{\theta}) D)}{1+R Q(\boldsymbol{\theta})}\right)\right\|_{2}^{2} .
$$

To deal with the above multiobjective optimization problem, we redefine $\varphi(t)$ and $y_{L}(t)$ in (18) as

$$
\begin{aligned}
\varphi(t) & =\left[-L_{M} \sigma \beta(t),-L_{T} \sigma(\beta(t)-D \tilde{n}(t))\right], \\
y_{L}(t) & =\left[L_{M}(\alpha(t)-X \tilde{r}(t)), L_{T}(\alpha(t)-X \tilde{n}(t))\right]^{\mathrm{T}},
\end{aligned}
$$

where $\tilde{n}(t)$ is a virtual reference such that $y_{0}(t)=T \tilde{n}(t), L_{T}$ is a filter selected as $L_{T}=$ $W_{T} T \Phi_{\alpha}(\omega)^{-1}$. The reference models for $G_{r y}$ and $G_{n y}$ are given by discretization of

$$
\begin{aligned}
M & =\frac{50^{2}}{(s+50)^{2}}, \text { and } \\
T & =\frac{50^{2}}{(s+50)^{2}}
\end{aligned}
$$

with the sampling time $T_{S}=1[\mathrm{~ms}]$.

The nominal controller stabilizing $P_{0}$ is evaluated from the relation

$$
M=\frac{P_{0} C_{0}}{1+P_{0} C_{0}}
$$

as

$$
\begin{aligned}
C_{0} & =\frac{M}{(1-M) P_{0}} \\
& =\frac{0.5036 s^{2}+68.52 s+4.110}{s(s+100)} .
\end{aligned}
$$

The weighting functions $W_{M}$ and $W_{T}$ are given to improve the tracking performance in low frequencies and the noise attenuation performance in high frequencies as

$$
\begin{aligned}
W_{M} & =\frac{200^{2}}{(s+200)^{2}}, \text { and } \\
W_{T} & =\frac{s^{2}}{(s+200)^{2}} .
\end{aligned}
$$


The Youla parameter $Q(s, \boldsymbol{\theta})$ is defined in the continuous-time so that the properness of $Q(s, \boldsymbol{\theta})$ and the relation, $Q \in \mathcal{R} \mathcal{H}_{\infty}$, are satisfied as

$$
\begin{aligned}
Q(s, \boldsymbol{\theta}) & =\frac{\theta_{1} s+\theta_{2} s^{2}+\theta_{3} s^{3}+\theta_{4} s^{4}+\theta_{5} s^{5}}{(0.06 s+1)^{5}} \\
& =\frac{1}{(0.06 s+1)^{4}}\left[\begin{array}{lllll}
s & s^{2} & s^{3} & s^{4} s^{5}
\end{array}\right]\left[\begin{array}{l}
\theta_{1} \\
\theta_{2} \\
\theta_{3} \\
\theta_{4} \\
\theta_{5}
\end{array}\right] \\
& =\sigma(s)^{\mathrm{T}} \boldsymbol{\theta} .
\end{aligned}
$$

The discrete-time Youla parameter $Q(z, \theta)$ is defined by discretization of $Q(s, \theta)$, i.e., $\sigma(s)$, with the sampling time $T_{S}=1[\mathrm{~ms}]$. In order to construct the type-I servo system even if the plant perturbs, the constant term of the numerator of $Q(s, \boldsymbol{\theta})$ is set to 0 such that $\left.Q(s, \boldsymbol{\theta})\right|_{s=0}=$ 0 in the continuous-time (Sakuishi et al., 2008).

\subsection{Experimental result}

The VRFT can be regarded as the open-loop identification problem of the controller parameter by the least-squares method. We select the pseudo random binary signal (PRBS) as the input for identification of the controller parameter as same as in the general open-loop identification problem, since the identification input should have certain power spectrum in all frequencies. The PRBS is generated through a 12-bit shift register (i.e., $T=2^{12}-1=4095$ samples), the reference signal $r_{0}$ is constructed by repeating this PRBS 10 times (i.e., $p=10, N=40950$ ). Firstly, we obtain the parameter $\hat{\boldsymbol{\theta}}_{\mathrm{w} / \mathrm{o}}$ as (26) when the stability constraint is not imposed.

$$
\hat{\boldsymbol{\theta}}_{\mathrm{w} / \mathrm{o}}=\left[\begin{array}{c}
-2.878 \times 10^{-2} \\
1.429 \times 10^{-2} \\
-1.594 \times 10^{-3} \\
1.184 \times 10^{-5} \\
1.339 \times 10^{-6}
\end{array}\right]
$$

Secondly, we obtain the parameter $\hat{\boldsymbol{\theta}}_{\mathrm{w} /}$ as (27) when the stability constraint is imposed.

$$
\hat{\boldsymbol{\theta}}_{\mathrm{w} /}=\left[\begin{array}{l}
1.263 \times 10^{-1} \\
1.261 \times 10^{-2} \\
7.425 \times 10^{-4} \\
4.441 \times 10^{-6} \\
4.286 \times 10^{-7}
\end{array}\right]
$$

The estimates of $\delta(\boldsymbol{\theta})$ for $Q\left(z, \hat{\boldsymbol{\theta}}_{\mathrm{w} / \mathrm{o}}\right)$ and $Q\left(z, \hat{\boldsymbol{\theta}}_{\mathrm{w} /}\right)$ are shown in Fig. 7. For $Q\left(z, \hat{\boldsymbol{\theta}}_{\mathrm{w} / \mathrm{o}}\right)$, the stability constraint is not satisfied around $60 \mathrm{rad} / \mathrm{s}$, and $\hat{\delta}\left(\hat{\boldsymbol{\theta}}_{\mathrm{w} / \mathrm{o}}\right)=7.424$. Since the sufficient condition for the robust stability is not satisfied, we can predict in advance of implementation that the closed-loop system might be destabilized if the Youla parameter $Q\left(z, \hat{\boldsymbol{\theta}}_{\mathrm{w} / \mathrm{o}}\right)$ was implemented. On the other hand, $\hat{\delta}\left(\hat{\boldsymbol{\theta}}_{\mathrm{w} /}\right)=0.9999$ for $Q\left(z, \hat{\boldsymbol{\theta}}_{\mathrm{w} /}\right)$, which satisfies the stability 


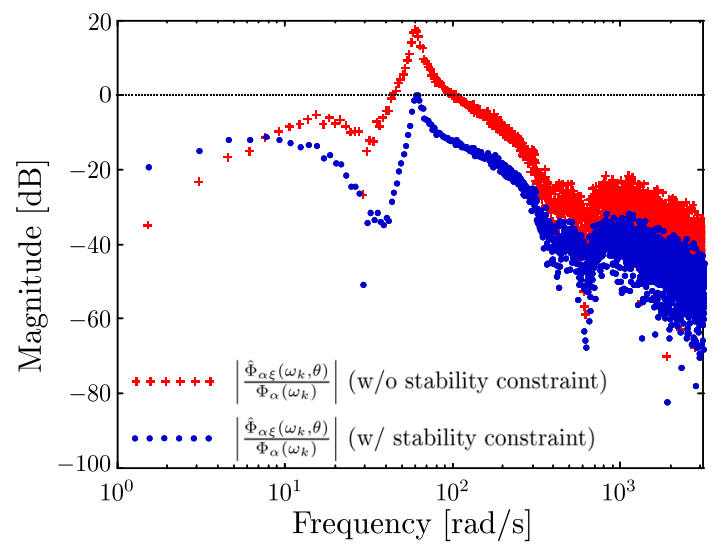

Fig. 7. Estimate of $\delta(\boldsymbol{\theta}), \hat{\delta}\left(\hat{\boldsymbol{\theta}}_{\mathrm{w} / \mathrm{o}}\right)$ and $\hat{\delta}\left(\hat{\boldsymbol{\theta}}_{\mathrm{w} /}\right)$.

constraint. Therefore, we can predict in advance of implementation that the closed-loop system could be stabilized if the Youla parameter $Q\left(z, \hat{\boldsymbol{\theta}}_{\mathrm{w}} /\right)$ was implemented.

Figure 8 shows the step responses of the GIMC structure with implementing $Q\left(z, \hat{\boldsymbol{\theta}}_{\mathrm{w} / \mathrm{o}}\right)$ and $Q\left(z, \hat{\boldsymbol{\theta}}_{\mathrm{w}} /\right)$. In the case of $Q\left(z, \hat{\boldsymbol{\theta}}_{\mathrm{w} / \mathrm{o}}\right)$, its response vibrates persistently, the tracking performance, $G_{r y}$, degrades compared with the case that the control system is governed by only the nominal controller $C_{0}$, i.e., $Q=0$. On the other hand, in the case of $Q\left(z, \hat{\theta}_{\mathrm{w}} /\right)$, its response does not coincides with the output of the reference model due to the long delay time, but Fig. 8 shows that the control system is at least stabilized. Moreover, we can confirm that the vibration is suppressed compared with the case of $Q=0$ and the proposed method provides the Youla parameter reflecting the objective function without destabilizing the closed-loop system. Although $J_{\mathrm{VR}}^{N}\left(\hat{\boldsymbol{\theta}}_{\mathrm{w} / \mathrm{o}}\right)<J_{\mathrm{VR}}^{N}\left(\hat{\boldsymbol{\theta}}_{\mathrm{w} /}\right)$, the response for $Q\left(z, \hat{\boldsymbol{\theta}}_{\mathrm{w} /}\right)$ is much closer to the output of the reference model than that for $Q\left(z, \hat{\boldsymbol{\theta}}_{\mathrm{w} / \mathrm{o}}\right)$. This observation implies

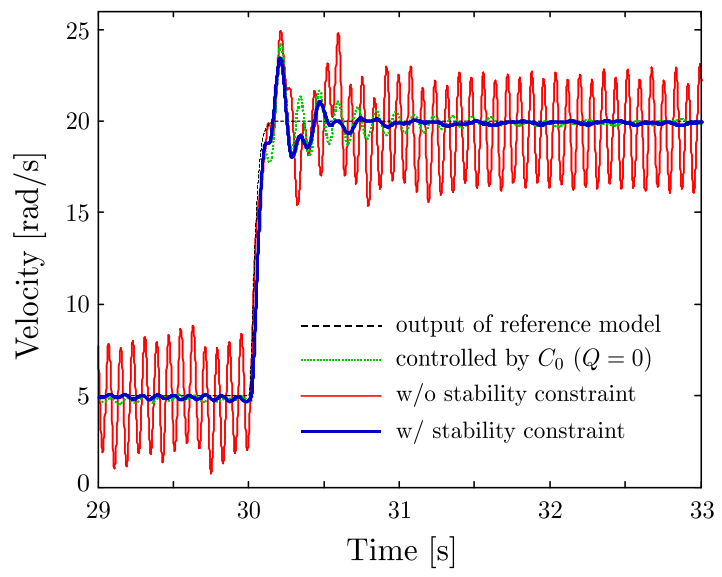

Fig. 8. Step responses for a belt-driven two-mass system with and without stability constraint. 
that only minimization of the 2-norm based cost function may not provide the appropriate stabilizing controller in model-free controller syntheses.

\section{Conclusion}

In this article, the design method of the Youla parameter in the GIMC structure by the typical model-free controller design method, VRFT, is proposed. By the model-free controller design method, we can significantly reduce the effort for identification of $R$ and the design of $Q$ compared with the model-based control design method. We can also specify the order and the structure of $Q$, which enable us to design a low-order controller readily. Moreover, the stability constraint derived from the small-gain theorem is integrated into the 2-norm based standard optimization problem. As a result, we can guarantee the closed-loop stability by the designed $Q$ in advance of the controller implementation. The effectiveness of the proposed controller design method is confirmed by the experiment on the two-mass system.

As a future work, we must tackle the robustness issue. The proposed method guarantees the closed-loop stability only at the specific condition where the input/output data is collected. If the load condition changes, the closed-loop stability is no longer guaranteed in the proposed method. We must improve the proposed method to enhance the robustness for the plant perturbation and/or the plant uncertainties. Morevover, though the controller structure is now restricted to the linearly parameterized one in the proposed method, the fully parameterized controller should be tuned for the higher control performance.

\section{References}

M.C.Campi, A.Lecchini and S.M.Savaresi: "Virtual Reference Feedback Tuning: a Direct Method for the Design of Feedback Controllers", Automatica, Vol.38, No.8, pp. 1337-1346 (2002)

D. U. Campos-Delgado and K. Zhou: "Reconfigurable Fault Tolerant Control Using GIMC Structure", IEEE Transactions on Automatic Control, Vol. 48, No. 5, pp. 832-838 (2003)

F. Hansen, G. Franklin and R. Kosut: "Closed-Loop Identification via the Fractional Representation: Experiment Design", Proc. of American Control Conference 1989, pp. 1422-1427 (1989)

K. van Heusden, A. Karimi and D. Bonvin: "Non-iterative Data-driven Controller Tuning with Guaranteed Stability: Application to Direct-drive Pick-and-place Robot", Proc. of 2010 IEEE Multi-Conference on Systems and Control, pp. 1005-1010 (2010)

K. van Heusden, A. Karimi and D. Bonvin: "Data-driven Controller Tuning with Integrated Stability Constraint", Proceedings of 47th IEEE Conference on Decision and Control, pp. 2612-2617 (2008)

H. Hjalmarsson, M.Gevers, S.Gunnarsson and O. Lequin: "Iterative Feedback Tuning: Theory and Applications", IEEE Control Systems Magazine, Vol.18, No.4, pp. 26-41 (1998)

A. Karimi, K. van Heusden and D. Bonvin: "Noniterative Data-driven Controller Tuning Using the Correlation Approach", Proc. of European Control Conference 2007, pp. 5189-5195 (2007)

L. Ljung: System Identification Theory for the User (second edition), Prentice Hall (1999) 
K. Matsumoto, T.Suzuki, S.Sangwongwanich and S.Okuma: "Internal Structure of Two-Degree-of-Freedom controller and a Design Method for Free Parameter of Compensator", IEEJ Transactions on Industry Applications, Vol.113-D, No.6, pp. 768-777 (1993) (in Japanese)

L. Mišković, A. Karimi, D. Bonvin and M.Gevers: "Correlation-Based Tuning of Linear Multivariable Decoupling Controllers", Automatica, Vol.43, No.9, pp.1481-1494 (2007)

M. Morari and E. Zafiriou: Robust Process Control, Prentice Hall (1997)

T. Sakuishi, K. Yubai and J. Hirai: "A Direct Design from Input/Output Data of Fault-Tolerant Control System Based on GIMC Structure", IEEJ Transactions on Industry Applications, Vol. 128, No. 6, pp. 758-766 (2008) (in Japanese)

M. Vidyasagar: Control System Synthesis: A Factorization Approach, The MIT Press (1985)

K. Yubai, S. Terada and J. Hirai: "Stability Test for Multivariable NCbT Using Input/Output Data", IEEJ Transactions on Electronics, Information and Systems, Vol. 130, No. 4 (2011)

K. Yubai, T.Sakuishi and J.Hirai: "Compensation of Performance Degradation Caused by Fault Based on GIMC Structure", IEEJ Transactions on Industry Applications, Vol.127, No. 8, pp. 451-455 (2007) (in Japanese)

K. Zhou and Z.Ren: "A New Controller Architecture for High Performance, Robust, and Fault-Tolerant Control", IEEE Transactions on Automatic Control, Vol.46, No.10, pp. 1613-1618 (2001)

K. Zhou and J. C. Doyle: Essentials of Robust Control, Prentice Hall (1998) 


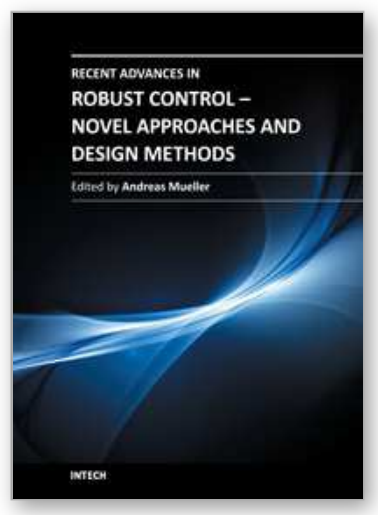

\author{
Recent Advances in Robust Control - Novel Approaches and \\ Design Methods \\ Edited by Dr. Andreas Mueller
}

ISBN 978-953-307-339-2

Hard cover, 462 pages

Publisher InTech

Published online 07, November, 2011

Published in print edition November, 2011

Robust control has been a topic of active research in the last three decades culminating in $\mathrm{H}$ 2/H_linfty and Imu design methods followed by research on parametric robustness, initially motivated by Kharitonov's theorem, the extension to non-linear time delay systems, and other more recent methods. The two volumes of Recent Advances in Robust Control give a selective overview of recent theoretical developments and present selected application examples. The volumes comprise 39 contributions covering various theoretical aspects as well as different application areas. The first volume covers selected problems in the theory of robust control and its application to robotic and electromechanical systems. The second volume is dedicated to special topics in robust control and problem specific solutions. Recent Advances in Robust Control will be a valuable reference for those interested in the recent theoretical advances and for researchers working in the broad field of robotics and mechatronics.

\title{
How to reference
}

In order to correctly reference this scholarly work, feel free to copy and paste the following:

Kazuhiro Yubai, Akitaka Mizutani and Junji Hirai (2011). A Model-Free Design of the Youla Parameter on the Generalized Internal Model Control Structure with Stability Constraint, Recent Advances in Robust Control Novel Approaches and Design Methods, Dr. Andreas Mueller (Ed.), ISBN: 978-953-307-339-2, InTech, Available from: http://www.intechopen.com/books/recent-advances-in-robust-control-novel-approaches-anddesign-methods/a-model-free-design-of-the-youla-parameter-on-the-generalized-internal-model-controlstructure-with-

\section{INTECH}

open science | open minds

\section{InTech Europe}

University Campus STeP Ri

Slavka Krautzeka 83/A

51000 Rijeka, Croatia

Phone: +385 (51) 770447

Fax: +385 (51) 686166

www.intechopen.com

\section{InTech China}

Unit 405, Office Block, Hotel Equatorial Shanghai

No.65, Yan An Road (West), Shanghai, 200040, China 中国上海市延安西路65号上海国际贵都大饭店办公楼405单元

Phone: +86-21-62489820

Fax: +86-21-62489821 
(C) 2011 The Author(s). Licensee IntechOpen. This is an open access article distributed under the terms of the Creative Commons Attribution 3.0 License, which permits unrestricted use, distribution, and reproduction in any medium, provided the original work is properly cited. 